\title{
Management of GIST: Perspective of Rajshahi Medical College Hospital
}

\author{
Uddin Md. Dayem ${ }^{1}$, Hannan A. B. M. Abdul ${ }^{2}$, Hasan Mohibul ${ }^{3}$
}

\begin{abstract}
A prospective study had been carried out between July 2003 and June 2010 in Radiotherapy Department of Rajshahi Medical College Hospital on the patients diagnosed as Gastrointestinal Stromal Tumor (GIST). A total of 32 patients were enrolled. Males 24(75\%) Predominated over the females $8(25 \%)$ with male female ratio 3:1. The patients were between 25 and 65 years with the mean age 45 years and majority (75\%) belonged to age above $\mathbf{4 0}$ years.

Common sites of involvement were ileum 14 (40.5\%), Stomach 12 (32.5\%) followed by duodenum 4 (15.25\%) and jejunum 2(11.75\%) in descending order. All patients attended after surgery except two who was inoperable. Patients with gross residual disease were planned for oral Imatinib mesylate $(400-600 \mathrm{mg} /$ day) as adjuvant therapy. The two inoperable cases and those with gross residual disease at primary or metastatic site were planned for palliative treatment. Imatinib mesylate $400 \mathrm{mg} / \mathrm{day}$ was planned for few cases. 3 cases with gross post operative residue were treated by 50Gys radiotherapy by conventional fractions from cobalt machine.
\end{abstract}

Among 20 cases who were planned for adjuvant therapy 4 completed their course of targeted therapy after four years and are on 2-3 monthly follow up are in disease free state - clinically and by investigation. Among the remaining-16 cases, two patients faced progression of the disease and died and two patients presented with liver metastasis and rest are on oral Imatinib therapy in escalating dose, now gradually improving.

Among the 12 cases, who were planned for palliative therapy, 4 post operative cases with gross residual mass, died due to disease progression. 2 post operative cases with gross residual disease, who received radiotherapy, did not come for subsequent follow up. Other 6 cases are yet on Imatinib and the tumor masses are regressing gradually.

TAJ 2010; 23(1): 1-5

\section{Introduction}

A gastrointestinal stromal tumor (GIST) is one of the most common mesenchymal tumors of the gastrointestinal tract (1-3\% of all gastrointestinal malignancies). They are typically defined as tumor whose behavior is driven by mutations in the Kit gene or PDGFRA gene, and may or may not stain positively for kit [1]
The term GIST was first introduced in 1983 by Mazur and Clerk to encompass gastrointestinal non-epithelial neoplasm that lacked immunohistochemical feature of schwann cells and did not have the ultra structural characteristics of smooth muscle cells1. GISTs continued to be rarely diagnosed until about the year 2000. At present

1 Professor \& Head, Department of Radiotherapy, Rajshahi Medical College, Rajshahi.

2 Professor \& Head, Department of Surgery, Rajshahi Medical College, Rajshahi.

${ }^{3}$ Assistant Professor, Department of Surgery, Rajshahi Medical College, Rajshahi. 
GISTs may by defined as morphologically spindle cell, Epitheloid or occasionally pleomorphic, Mesenchymal tumors that usually arise from gastrointestinal tract express the KIT protein and that often harbour mutation of a gene that encodes for a type III receptor tyrosine kinase. The KIT protein is readily detectable by immunohistochemical photochemical assay and the gene mutation can be detected by mutation analysis usually based on DNA sequencing. Other useful diagnostic features for GIST are negative immnunostainig for desmin (95\%) and absence of lymph node and lung metastasis (95\%) 2.

GISTs vary in malignancy potential ranging from small, incidentally detected tumors with excellent outcome to aggressive sarcomas2. The proportion of overtly malignant or high -risk GISTs is 2035\%of all GISTs 3.4 suggesting that the annual incidence of GISTs with a high malignancy potential is about 5 per million. It is likely that many small GISTs are not reported to cancer registries. The frequency of GISTs may change with time because of evolving diagnostic criteria and the greater awareness 5 .

GISTs might constitute approximately one sixth to one third of all sarcoma. It may originate anywhere in the gut. Stomach (40-60\%) and small intestine (30-40\%) are the most common locations. Colon, rectum oesophagus and retro other intra- abdominal organs are other sites of origin. Previously only a few cases of GISTS have been reported. This series is the largest series in our country which might reflect the real picture of GISTS prevailing amongst the people of our country.

Although the exact incidence is still somewhat unclear it is now estimated the 5,000 people each year develop GISTs.

About $40-70 \%$ of GIST s arise from the stomach, $20-40 \%$ arise from the small rectum. GIST s can also be found in the oesophagus $(<5 \%)$. Sometimes GISTs are found in abdominal cavity.

\section{Material and Methods}

This prospective study has been carried out between July 2003 and June 2010 in the Department of Radiotherapy, Rajshahi Medical College Hospital. Patients diagnosed as GIST referred from other departments, clinics, hospitals were included in this study.

Detail history was taken, thorough physical examination was done and related investigations (chest X-ray, Ultrasonography of whole abdomen, CT scan of whole abdomen (in selected cases ) were done for staging purpose. Routine blood test, liver and renal functions were done in all cases before starting the treatment.

All patients except two (who were inoperable) attended after surgery (total resection / debulking). Patients with no gross residual disease were planned for oral imatinib mesylate as adjuvant therapy. Inoperable and those with gross residual disease (primary / metastasis site) were planned for palliative treatment.

At the end of the treatment all the patients were planned for two to three monthly follow up. Thorough physical examination, all routine investigations such as routine blood test, liver and renal functions tests, chest X-ray, ultrasonography of whole abdomen, CT scan of abdomen (selected cases) were done. All the information was documented in the individual printed sheets for compilation.

\section{Results}

Among the 32 patients studied, 24 (75\%) were male, 8 (25\%) were female and the ratio of male female were $3: 1$, which indicates male predominance.

Patients aged between 25 and 65years with mean age 45years.

Table-1: Distribution of patients according to age $(n=16)$

\begin{tabular}{lcl}
\hline Age in years & No of patient's & Percentage \\
\hline $21-30$ & 6 & $18.75 \%$ \\
$31-40$ & 2 & $6.25 \%$ \\
$41-50$ & 6 & $18.75 \%$ \\
$51-60$ & 14 & $43.75 \%$ \\
$61-710$ & 4 & $12.5 \%$ \\
\hline
\end{tabular}


Table-2: Distribution of patients according to printing feature $(n=16)$

\begin{tabular}{lcl}
\hline Presenting feature & $\begin{array}{c}\text { No of } \\
\text { patient's }\end{array}$ & Percentage \\
\hline Abdominal lump & 32 & $100 \%$ \\
Abdominal pain & 32 & $100 \%$ \\
$\begin{array}{l}\text { Distention of } \\
\text { abdomen }\end{array}$ & 6 & $18.75 \%$ \\
Vomiting & 8 & $25 \%$ \\
Leg Oedema & 2 & $6.25 \%$ \\
Low back pain & 2 & $6.25 \%$ \\
Weight loss & 32 & $100 \%$ \\
Anorexia & 32 & $100 \%$ \\
P/R bleeding & 4 & $12.50 \%$ \\
Fever & 2 & $6.25 \%$ \\
\hline
\end{tabular}

Table-3: Distribution of patients according to site of tumors $(n=16)$

\begin{tabular}{lcl}
\hline Sites & No of patient's & Percentage \\
\hline Ileum & 12 & $37.5 \%$ \\
Stomach & 10 & $31.25 \%$ \\
Colon & 6 & $18.75 \%$ \\
Duodenum & 2 & $6.25 \%$ \\
Jejunum & 2 & $6.25 \%$ \\
\hline
\end{tabular}

All patients except two (who were inoperable) attended after surgery (total resection / debulking).Ten patients with no gross post operative residual disease were planed for oral imatinib mesylate as adjuvant therapy. Dose of imatinib were $400 \mathrm{mg}$ /day for four to five years. One inoperable and five cases with gross post operative residual disease (primary /met static site) were planned for palliative treatment. Imatinib, mesylate 400mg/ day was planned for five cases. One case with gross post operative was treated by 50 Gys radiotherapy by conventional fractions from Cobalt machine due to financial reason.

Among 20 cases who were planned for adjuvant therapy 4 have completed their course of targeted therapy after four years and are on 2-3 monthly follow up and in disease free state - clinically and by investigation. Among the remaining 16 cases, 2 patient faced progression of the disease and died and 2 patient presented with liver Metastases and rest are on oral Imatinib in escalating dose, now gradually improving Others are on adjuvant therapy without any clinically detectable disease.

Among the 12 cases who were planned for palliative therapy, 4 post operative case with gross residual diseases died due to disease progression. 2 post operative case with gross residual disease, who received radiotherapy, did not turn up after completion of radiotherapy for subsequent follow up. Other 6 cases are yet on Imatinib and the tumour masses are regressing gradually.

No patient had Grade III or IV toxicity. Among all patients 5(31.25\%) showed Grade I or II toxicity and managed conservatively.

\section{Discussion}

Gastrointestinal stromal tumor is a recently recognized tumor entity ${ }_{2}$. In the past these were classified as leiomyoma, leiomyoblastoma and leiomyosarcoma as they resemble histological to these smooth muscle neoplasms 6,7 . These tumors were also noted to be particularly resistant to standard chemotherapy regimens used to treat leiomyosarcoma elsewhere. Another difference is that a significant subset of these was noted to lack the characteristics muscle antigen ${ }_{8}$. In1983 Mazur and Clark introduced the generic term GIST $_{1}$. However the term GIST was still controversial and not fully specific in its definition ${ }_{8}$. Other terms were generated based on the fact that neural crest antigens such as neuron specific enolase and S100 could be demonstrated in Gusts cells, which led to the terms plexosarcoma and Gastrointestinal autonomic nerve tumor ${ }_{9,10}$.

These tumors have certain histological similarities to the interstitial cell of Cajal of GI tract ${ }_{11}$. Both express the KIT receptor tyrosine kinase and share a common precursor cell ${ }_{12,13}$. Hitora et all in 1998in Japan reported the relationship between GIST and certain mutation in the kit proto-on gene that conferred uncontrolled activation to the lit signaling enzyme and this cause neoplastic growth of the cells ${ }_{14}$.

The median age at diagnosis is 66-69 years ${ }_{2}$. In SEER registry data the median age was 63 years 15 . In our study, the age range was from 25to 65 years. Peak incidence occurs at the age of $6^{\text {th }}$ decade which correlate with the SEER data. Only $\sim 3 \%$ of GISTs are diagnosed before the age of 21 and rarely diagnosed in children ${ }_{2}$. Our study correlates with that as on patient was below 25years. 
No predisposing factors have been described in relation with $\mathrm{GIST}_{2}$. Here no predisposing factors are detected.

In the GISTR series $55 \%$ of GISTS occurred in male15 whereas equal gender distribution was found in a population based analysis from Sweden as well as in a large series in Korea ${ }_{13,17}$. In this series the numbers of male and female patients are 24 and 8 respectively with a male female ratio 3:1 indicating male predominance which resembles to SEER series.

The most common symptom at presentation is bleeding $_{16}$. Large GISTS often protrude from the site of origin and grow between the bowel loops and the abdominal organs and may also erode gastrointestinal tract lumen. Bleeding may occur rather into the abdominal cavity causing acute abdominal pain and severe anaemia or into the gastrointestinal tract lumen causing haematemesis, melaena and anaemia ${ }_{2}$. Patients may present with various other symptoms like abdominal pain or discomfort, early satiety, obstructive jaundice, dysphasia, fever and anaemia related fatigue \& palpitation $_{16}$. They may present with an abdominal tumour with no symptoms2. Between $10-20 \%$ of patients present with metastasis disease 3,15 .

In our series all the patient (100\%) presented with abdominal lump, abdominal pain and weight loss 6 (18.75\%) and anorexia, patients presented with abdominal distention 8 25\%), patients presented with vomiting, 2 6.25\%) Patient had leg oedema, (6.25\%) had low back pain, 2 (6.25\%) had fevers but only $4(12.50 \%)$ patients presented with per rectal bleeding.

In SEER data 51\% cases arose from stomach $36 \%$ from small intestine, 7 \%from colon 5\%from rectum and $1 \%$ from oesophagus15. Here Common sites of involvement were ileum 14 (40.5\%), Stomach 12 (32.5\%) followed by duodenum 4 15.25\%) jejunum 2 11.75\%) in descending order ${ }_{17}$.

Most ( 95\%) GISTs are CD117 antigen positive3, 4. In CD117 positive cases PKC-0 is highly expressive. In our series, 15 93-75\%) cases were CD117 positive which correlates with above data. one patient could not do CD 117 due to financial constrain.
Surgery is the standard treatment for GISTs 2. Administration of adjuvant imatinib mesylate appears attractive to prevent recurrence. Patients who have tumor rupture in the abdominal cavity and those rendered free from metastasis by surgery face a very high risk of recurrence and should be considered as candidate for Imatinib therapy. The optimal duration for administration is unknown. Adjuvant radiation and conventional chemotherapy had on proven value and were not recommended. Regarding treatment of our patients, 13 (93.75\%) undergone surgery and planned for oral Imatinib mesylte (400-600ng/day) and those with residual disease (primary/metastasis site) were planned for palliative treatment.

Adverse effects of Imatinb mesylate therapy are usually mild to moderate. The most common adverse effects are oedema (usually peiorbital) muscle cramps in fingers and feet, diarrhea, nausea, vomiting, fatigue and $\operatorname{rash}_{2}$. Anaemia, neutropenia elevation of liver transaminase levels are also common.

None of the patient suffered from grade III or IV toxicities. Grade I and II toxicities are managed and did not hamper the treatment schedule.

Among 20 cases who were planned for adjuvant therapy, 4 patients had Imatinib for four years and then stopped. Till date they are free from any disease and on two monthly follow up. 2 patients died due to disease progression, 2 patients presented with liver metastasis after 3years, receiving oral Imatinib in escalating dose, now gradually improving. Remaining 12 cases are getting imatinib and are asymptomatic without progression of disease. Among the 12 cases who were planned for palliative therapy, 4 post operative case with gross residual mass died due to disease progression. 2 post operative cases with gross residue who received radiotherapy, absconded after completion of radiotherapy and his fate is not known to us. Other 6 cases are yet on imatinib and the gross masses are regressing gradually.

No patient had Grade III or IV toxicity. Among all patients 10(31.25\%) showed Grade I or II toxicity and managed conservatively 


\section{References}

1. Mazur Mt, Clark HB. Gastric stromal tumors reappraisal of histogenesis. Am J Surg pathol 1983; 7:507-519.

2. Joensuu H. Gastrointestinal stromal tumor (GIST) Annals of Oncology, September, 2006; vol-17:280-286.

3. Nilsson BP, Bumming P, Meis-Kindblom JM et al Gastrointestinal stromal tumors: The incidence, prevalence, clinical course and prognostication in the preimatinib mesylate era. Cancer 2005; 103:n821-829

4. Miettinen $\mathrm{M}$, Lasota. Gastrointestinal stromal tumors- definition, clinical, histological, immunohistochemical and molecular genetic features and differential diagnosis. Virchows Archiv 2001; 438:1-12.

5. Goettsch WG, Bos SD, Breekveldt-postma N et al. Incidence of gastrointestinal stromal tumors is underestimated: Results of a nation- wide study Eur $\mathrm{J}$ cancer 2005;2868-2872.

6. Golden T, Stout AP. Smooth muscle tumors of the gastrointestinal tract and retroperitoneal tissues. Gynecol Obstet 1941; 73:784.

7. Stout AP Bizarre smooth muscle tumors of the stomach. Cancer 1962; 15:400.

8. Demetri G.D. Gastrointestinal stromal tumors.De Vita V.T, Hellman S,rosenderg S.A, Devita's Cancer Principles \& practice of Oncology, $\mathrm{Vol}-2,7^{\text {th }}$ ed: 1050-1059.

9. Herrera GA, Pinto de moraes H, Grizzle WE, et al, malignant small bowel neoplasm of enteric plexus derivation (plexosarcoma).light and electron microscopic study confirming the origin of the neopasm. Dig Dis Sci 1984; 29:275.
10. Walker p, Dvorak AM. Gastrointestinal autonomic nerve (Gan) tumor. Ultastrctural evidence a newly recognized entity. Arce Pathol Lab Med 1986; 110:309.

11. Perez-Atayde AR, Shamberger RC. Kozakewich HW. Neuroectodermal different ion of the Gastrointestinal tumors in the carney triad. An ultrastructural and immunohistochemical study. Am J surg pathol 1993; 17:706.

12. Kindblom LG, remotti HE, Aldenborg $F$, et al Gastrointestinal pacemaker cell tumor (GIPACT) : gastrointestinal stromal tumors show phenotypic characteristics of the interstitial cells of Cajal .Am J Pathol 1998;152:1259.

13. Sircar k, Hewlett BR, Huizinga JD, et al Interstitial cells of Cajal as precursors of gastrointestinal stromnal tumors. Am J Surg pathol 1999; 23:377.

14. Hirota S, Isozaki K, Moriyama $\mathrm{Y}$, et al - of function mutations of c-kit in human gastrointestinal stromal tumors. Science 1998; 279:577580.

15. Tran T, Devila JA, El-Serag HB. The epidemiology of malignant gastrointestinal stromal tumors: an analysis of 1458 cases from 1992to 2000.Am J Gastroenterol 2005;1 00:162-168.

16. Miettinen M, Sobin LH Lasota J. Gastrointestinal stromal tumors of the stomach: A clicpathologic, immunohistochemical and molecular genetic study of 1765 cases with long term follow up. Am J surg pathol 2005; 29:52-68.

17. Kim Km, Kang DW. Moon WS et al. Gastrointestinal stromal tumors in Koreans: Incidence and the Clinical, athological and immunohistochemical findings. J Korean Med Sci 2005; 20:977-984. 